\title{
From Biodata to Somadata
}

\author{
Miquel Alfaras ${ }^{1,2}$, Vasiliki Tsaknaki ${ }^{3}$, Pedro Sanches $^{3}$, Charles Windlin $^{3}$, \\ Muhammad Umair ${ }^{4}$, Corina Sas ${ }^{4}$, Kristina Höök ${ }^{3}$ \\ PLUX Wireless Biosignals ${ }^{1}$ \\ Lisbon, Portugal \\ Universitat Jaume $\mathrm{I}^{2}$ \\ Castelló de la Plana, Spain \\ malfaras@plux.info \\ KTH \\ Royal Institute of Technology ${ }^{3}$ \\ Stockholm, Sweden \\ \{tsaknaki,sanches, windlin,khook\} \\ @ kth.se \\ Lancaster University 4 \\ Lancaster, United Kingdom \\ \{m.umair7, c.sas\} \\ @ lancaster.ac.uk
}

\begin{abstract}
Biosensing technologies are increasingly available as offthe-shelf products, yet for many designers, artists and nonengineers, these technologies remain difficult to design with. Through a soma design stance, we devised a novel approach for exploring qualities in biodata. Our explorative process culminated in the design of three artefacts, coupling biosignals to tangible actuation formats. By making biodata perceivable as sound, in tangible form or directly on the skin, it became possible to link qualities of the measurements to our own somatics - our felt experience of our bodily bioprocesses as they dynamically unfold, spurring somatically-grounded design discoveries of novel possible interactions. We show that making biodata attainable for a felt experience - or as we frame it: turning biodata into somadata - enables not only first-person encounters, but also supports collaborative design processes as the somadata can be shared and experienced dynamically, right at the moment when we explore design ideas.
\end{abstract}

\section{Author Keywords}

biosensing; soma design; first-person perspective; affective technology; interaction design;

\section{CCS Concepts}

-Human-centered computing $\rightarrow$ Interaction design theory, concepts and paradigms;

\section{INTRODUCTION}

In the past decade, biosignals found many applications in healthcare and HCI [42]. They allowed for applications that support self-awareness by displaying properties of our bodies as they unfold in real-time, or applications that are able to track one's mood over certain periods of time [18], or even to assess one's health status [3]. On the other hand, design researchers and artists bring creative potential to the field,

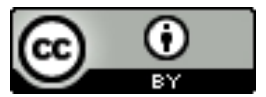

This work is licensed under a Creative Commons Attribution International 4.0 License.

CHI '20, April 25-30, 2020, Honolulu, HI, USA.

(C) 2020 Copyright is held by the author/owner(s). ACM ISBN 978-1-4503-6708-0/20/04.

http://dx.doi.org/10.1145/3313831.3376684 driving further development of biosensing technologies to fit outside the narrow settings they were originally designed for [59, 24, 25].

But how can one engage creatively with biosensing technologies in a design process, and how to approach biodata as a design material to work with? In an attempt to find a path to better align with the designerly processes employed outside purely medical or technology-driven explorations with biosensing technologies and biodata, we decided to engage with a design methodology named soma design [20]. Soma design puts a first-person, aesthetic and sensuous experience and expertise in the front seat during the design process [21]. Through engaging with and deepening one's capacity to discern sensuous somatic experiences, one can "examine and improve on connections between sensation, feeling, emotion, subjective understanding and values"[28]. A soma design process thrives off the "aesthetic potential of the sociodigital materials and the creative process of shaping these into dynamic gestalts and orchestrated experiences'([20], p.127). We saw a potential in soma design to explore biodata and make it available as a sociodigital material, motivating the work presented here.

We present design explorations resulting from soma designfocused workshops, where we introduced biosensing technologies to a range of participants, including designers who had not been much exposed to biosensing prior to the workshops. Through our explorations we found that carefully coupling biodata to different actuation modalities was a fruitful way to generate design ideas based on biodata. In particular, we found that certain couplings of biodata to actuation worked better for collaboratively experiencing and creating meaning from the biodata - generating what we frame as somadata - somatically experienced data. Somadata 1) allows for first-person, felt, somatic experiences of biodata; 2) allows for collaborative experiences, in turn leading to collective meaning-making and opening for novel design ideas; and 3) does not constrain, physically or otherwise, the designerly explorations of body dynamics of relevance to what is being designed.

While reflecting on our process, we started thinking more deeply about data and what it represents. When it pertains to our own bodies, biodata can show us fragments of complex processes, like when heart rate speeds up because we are 
afraid, or because we are in love. Making biodata available as a sociodigital material means taking into account how our bodies and minds together - our somas - make sense of the world. This is why we refer to the materialisation of biodata to make it tangible and felt in the design process as turning data into somadata.

We end by discussing potentials and limitations of our process, and how these highlight paths for future work around designing with biodata in interaction design. The main contributions of this work are: a) a novel approach to spurring creative exploration of biosensing in early ideation design phases, arising from engaging with soma design methods. This approach involves first exploring different ways to couple biosensors to different actuation forms that can then later be explored and experienced with the design team. These couplings are key to enable first-person engagements with biodata that in turn can spur co-exploration and articulation of design discoveries, carefully grounded in somatic experiences, and b) seeking and finding those interactions that let biodata be turned into somadata of relevance to the design challenge at hand. We present three specific examples of somadata couplings that other researchers may find inspiring when designing for and with bodily data.

\section{BACKGROUND}

At a fundamental level, biosignals are time representations of changes in energy produced in the body [47]. These changes correspond to energy variations of different origin such as in electrical, chemical, mechanical and thermal processes. While affordable costs motivated the proliferation of biodata-based applications in everyday life $[7,46]$ the design of biosignalbased interactions beyond biomedical paradigms still remains much unexplored. When designing with biosignals, several aspects need to be taken into account.

\section{Exploring modalities}

A fundamental aspect when designing with biosensing comes to choosing appropriate types of biodata to work with. For example, a designer wishing to measure arousal might choose to look into skin conductance or heart rate, blood pressure or movement [41] as possible ways of approximating how aroused users are. But different modalities will capture different aspects of the experience being measured, and consequently will open up different design directions and pose different constraints.

\section{Exploring sensing mechanisms}

Once a modality is chosen it can in turn be captured by different sensing mechanisms. For example, movement can be captured by wearable sensors or by a camera, among many other possibilities. Moreover, sensors come at many different fidelity levels. Some sensors might easily break or they might be hard to attach to the body or to clothing. For example, in the work by Fdili Alaoui [14], sensors are used as part of a dance performance and, as she explains, some sensors were not made for the extreme movement and profuse sweating dancing entails. That is, as a designer, one needs to understand exactly what use scenario a sensor is made for. Fdili's position is that this "technology resistance" should not be seen as a problem, but as a path to spur artistic creativity. Still, her initial assumption on what biosensing could potentially contribute to the dance performance was not met - an insight that did not, and indeed could not, arrive solely from reading the technical specification of the sensor.

\section{Exploring sensor placements}

Sensors have to be attached to the right place on the body in order to capture the intended biosignals. For example, Tsiamyrtzis et. al [57] analyzed different body locations, including wrist, finger, palm and sole for Electrodermal Activity (EDA) sensing, and found some locations to be very good in terms of signal quality while in other locations the acquisition of EDA data were quite challenging. As pointed out in by Can and colleagues [10], gathering biosensing data outside the laboratory setting is notoriously difficult as body movements might interfere with the signal we wish to measure.

\section{Processing biodata}

Even when designers pick the right sensor for gathering specific biodata and place it correctly on the body, the signal itself needs to be processed in order to pick out the right features of relevance to the application at hand. Our bodily processes are not always aligned, meaning they are not always telling one singular story about what is happening. And in fact, there is not even a single story that can account for all the different processes taking place in and around our bodies at the same time [36]. For example, a facial expression might express happiness according to some facial recognition algorithm, at the same time as the hormonal levels in the bloodstream or sweat on the skin might indicate stress or fear. Any application built on one or several biosignals must therefore carefully account for the complexities of our somas, even allowing for ambiguous meaning-making to open the space for interpretation, as otherwise, it will be reductionist account of what is going on.

\section{Sensemaking}

Finally, one must also consider how biodata or events derived from biosensors come to be integrated in the everyday lives of those we design for. First, users often ascribe too much precision and authority to biodata-based systems [24]. Sometimes even to the point where they trust the system more than their own experiences. This put high demands on designers to properly convey the limitations of what the system 'knows'. Second, users may have preconceived ideas that colour their meaning-making processes. In a study lasting for a month Sanches and colleagues [40] observed that different user groups came up with entirely different interpretations of an EDA-enabled (Electrodermal Activity), real-time, monitoring system. Participants involved in sports assumed that they would be able to see recovery patterns through their biodata, while people suffering from stress issues looked for indicators of stress or emotion patterns. Others considered the system mainly as a life-logging system. Depending on their preconceived assumptions of what they would be able to see in the data, they did not even see data that spoke against their preconceptions.

Lastly, users also actively combine different systems and appropriate them for their own settings [9]. Rooksby and colleagues [39], studied how people use various tracking devices as part of their everyday life, and concluded that we cannot 
expect "people to act as rational data scientists". Users track their data on and off, they switch between devices, and they engage with their data more in periods when they have a clear need to do so.

\section{Turning Biodata into a Design Material}

There are of course different paradigms for how to design systems that rely on biodata. For example, a biomedical engineering paradigm [48] aiming at making advanced healthcare systems that diagnose or monitor diseases has other requirements than a designerly, explorative paradigm. In contrast to such engineering paradigms, designers start from ill-defined problems and use sketching and material explorations in order to arrive at different solutions [11].

In HCI there is a recent turn to exploring biodata as a "material" that can be made available to designers. Data, algorithms, wireless connectivity and other "immaterial materials" [50] are seen as materials with their own affordances and properties that one can design with $[62,15,38,45,61]$. By considering data as a material and translating its "immaterial" properties into tangible form it can be made accessible in, for example, the ideation stages of a design process (e.g.[12, 13]). Touching, feeling and interacting with the material affordances is important for grounding design work in what is possible, but also for spurring novel ideas arising from the technology itself. But as argued by Umair and colleagues [59], exploring sensor data as a material and translating such data in a material form by coupling sensing to actuation has only been partially explored and more work is needed.

\section{THEORETICAL BACKDROP: SOMA DESIGN}

To properly understand how to turn biosensing data into a form that can be felt, we turned to Soma Design. In a soma design paradigm, it is argued that the experiences of designers' first-person experiences, their lived bodies, is where design work originates. Let us briefly introduce some of the core concepts and practices underpinning soma design, as these directed our research method.

\section{First-person Perspective}

First-person is a concept from Merleau-Ponty's Phenomenology of perception [34]. Merleau-Ponty makes a distinction between the first-person perspective on the body, the lived body, the body through which we live our lives and experience the world, and the third-person perspective of seeing the body as an object in the world $[21,52,53]$. In addition to the first person and the third person perspectives on the body, there is also the interpersonal second person perspective. It involves emphatically experiencing the body of another. The second person engagement can be seen as a process of perceiving and engaging in an intercorporeal sense $[66,34]$, that is knowing someone else's experience by mirroring their expressions through your own. As pointed out by Svaneaes [53], this will always be difficult as you cannot fully know the experience of someone else. But we might also argue that knowing your own experience is also a process: you have to turn inwards, observe and interpret [33].

In design work, using your own subjective stance as a resource to ground design decisions is a recognised method
$[35,32]$, even if still seen as quite controversial as it does not guarantee that the design will be of use to anyone else. This subjective stance leverages designers' emotions [43, 44] and personal experiences [67]. Overall, a first-person design stance breaks with the user-centred processes advocated in $\mathrm{HCI}$. And of course, while we argue that the felt experience is key to designing with biosensing data, it does not liberate us from engaging with the needs of users at various points in the design process.

\section{Soma-centred Practices}

To arrive at a position where we, as designers, can engage in lived experiences of biodata and use those to shape novel designs, we first have to train our aesthetic sensitivities. By repeatedly engaging with different body practices or touching, feeling, and probing the qualities of different digital materials or applications, we can develop the ability to differentiate between small nuances in the somatic responses to different interactions [20]. This resembles artistic practices in which artists always start by learning about their materials before they can mould them into artistic expressions.

There is a set of underlying tactics (employed in many bodily practices) helping us discern what is going on in a dynamically unfolding experience and attend to its structure and all the details involved. One is to make the habitual "strange" in order to make it clearer to us $[63,31]$. Imagine, for example, unlocking your door with your other, non-habitual, hand and how that allows you to really deconstruct what is going on: entering the key into the lock, turning the key, opening the door and pulling out the key. Deconstructing movements like this underpins design methods, where materials and interactions are done in unusual manners in order to explore what could be [20]. Novel design ideas arise from doing habitual movements or experiencing design materials in the non-habitual, strange, way.

A second path to somaesthetic design awareness is to focus more intently on one of your senses or one body part in order to attend properly to what is happening. In particular, it might be important to experience, e.g. technological materials blindfolded, as the visual sense is a dominant sense in humans. A third path to somaesthetic design proficiency, is to guide your awareness to different parts of an experience by subdividing and structuring the senses, different parts of the body, and movements involved. Such a method was used in by Lee and colleagues [30] in interaction design contexts, for bringing attention to the soma.

As our somaesthetic expertise increases, it is also sometimes beneficial to be able to put words to what we experience. A richer language allows us to share and point to specific experiences that we may later want to pick up on in our design processes [23]. This language does not necessarily have to be verbal. Inspired by the work by Khut [28], we used body sheets to document our experiences. In the same spirit as [56, 65], by filling in a body sheet before and after experiencing a bodily exercise or touching/feeling/probing a technological material, we could compare what effects these have on us. Body sheets let us share our experiences with one-another in the design team, as well as with any end-users we invite into 
our design explorations. Structured tactics like these bring rigour to a practice that might otherwise seem quite mysterious and ineffable [8].

\section{RESEARCH PROCESS AND DESIGN STAGES}

The findings reported in this paper are a result of 4 distinct research collaborations, over the course of 8 months, involving 4 host institutions in different countries. Each collaboration involved different sets of participants and particular aims and outcomes, but all shared the overarching soma design methodology. All participants provided signed consent forms at the different workshops, informing about our research and publication scope. Our explorations were driven by the idea that designing slowly, disrupting the habitual and engaging with designers' feelings and passions would render novel design ideas for biosensing-mediated interactions. However, we did not know beforehand which activities would best facilitate the meeting between designer and biosensing material - and we explored several different paths before coming to one that rendered the most success. Each collaboration included creative design workshops, prototyping sessions before and after each workshop, as well as research explorations in our lab. After each workshop we reflected on and progressively refined the ways by which we introduced and explored biosensing technologies to other designers, culminating in what we frame as somadata.

The authors of this paper participated in, and sometimes ran the workshops that we present in all the stages described in the following sections. Let us provide a brief account of each of these stages to show how the ideas on how to introduce biosensing into creative workshops grew from one stage to the next.

\section{Stage 1: Engaging in First-Person Actuation}

In the first stage of our research process, a design workshop prompted our whole focus on first-person perspectives to articulate felt bodily sensations. This workshop was part of a five-day training event for $\mathrm{PhD}$ students designing for affective well-being [1]. There were 22 participants in total, including $14 \mathrm{PhD}$ students from different disciplinary backgrounds such as interaction design, computer science, psychology, engineering, and industrial design. Some authors were organisers of this event. The aim of this event was to learn about soma design as a methodology. Inspired by Tsaknaki and colleagues [56], we ran several sensitising activities, including Contact Improvisation, Feldenkrais exercises, and body scan activities, as well as more design-oriented activities such as a Magic Machines exercise [4] and an Aesthetic Laboration (A-lab) workshop facilitated by Akner-Koler [2]. The latter was particularly important for how we developed the concepts in this paper.

An A-lab is a structured method for interacting with physical and technical materials with the aim of increasing one's aesthetic sensitivity, and the ability to feel and articulate different bodily sensations and properties of materials. It might be the softness of fur, coldness of metal, or the buzzing of a vibration motor. The participants were divided into groups of three, and following Akner-Koler's structured method [2] , explored, shared, documented, and presented to others their differing, but also similar, first-person experiences. The A-lab was focused on actuation, but it spurred the idea that it should be possible to explore biosensing in a similar way, foregrounding first-person experiences, sharing and articulation of such experiences, and a focus on the somatic experiences.

\section{Stage 2: From Actuation to Biosensing}

Influenced by the A-lab from the first stage, we developed a similar structured method for exploring aesthetic properties of biosensors and biodata in groups. We ran two design exploration sessions at KTH University in Stockholm, in the form of short, structured workshops. Each session, lasting approximately three hours, was ran by the first author, and attended by eight participants: four graduate students and four researchers (including the second and third authors), with backgrounds in interaction design and engineering. All participants were familiar with the soma design method and/or had been involved in Stage 1.

In both sessions, participants worked in pairs, and each pair was given a laptop and several biosensors to explore: Electrocardiography (ECG), Electromyography (EMG), Breathing, Accelerometer (ACC), or Electrodermal Activity (EDA). We used the BITalino [7] biosensing platform, which comes with a software for real-time signal acquisitions, visualised real-time on the laptop screen. Following a soma design method, at the beginning of each session participants engaged in a body scan in order to focus on and articulate sensations in their bodies, before embarking on the biosensing explorations. One person was facilitating the placement of a biosensor on the experiencer's body, while both were looking at the screen, where real-time visualisations of biodata were depicted as a graph. Participants were encouraged to verbalise and share thoughts and understandings with one another.

Here we provide a short description of how two participants explored the EMG sensor. Participant A placed the sensor on participant's B cheek. Both then turned to the visualised biodata on the screen, following how B performed different slow or fast - movements with his mouth. Participant A adjusted the placement of the biosensor on the face of participant $\mathrm{B}$, to better capture the movements of the muscles. While looking at the graph, participant A said: "the signal seems unstable. Can you try to repeat exactly the same movement, to see whether there is s repetition in the depicted signal?". B responded: "Before, when the electrodes were placed closer to my ear, the signal seemed more robust-But still, if I move my lips very little, there is this strange peak on the graph that I do not know where it is coming from. Do you see it?" - pointing to the screen for B to see the peak.

Similar to an A-lab, our aim was to highlight rich properties and deeper aesthetic appreciation for how different ways of moving could be seen in different types of biodata. In that way the properties of a biosensor would be exposed, and at the same time the intersubjective articulation and meaning-making of biosensing representations, happening between two people, would contribute to a deeper understanding of a biosensor's properties in relation to specific placements of the sensor on the body. This stage stressed the need to engage with our 
whole somas - not with a biosignal representation as an abstract signal isolated from the body. Looking at the signals on a screen was helpful for getting a basic understanding of how a sensor works and which data it senses. But at the same time, the need to explore sensor data in other modalities emerged, aligned with other bodily parts or senses.

\section{Stage 3: Designing for Balance}

In the third stage we introduced biosensing into a creative workshop aiming to explore designing for "balance"- both how to keep balance, but also how to disrupt and introduce balancing challenges. Similar to the work done in the first stage, participants engaged in sensitising bodily activities aiming at reflecting how we balance ourselves when e.g. walking on a balancing rod or dance with a prosthetic limb. The workshop was organised by our research group in collaboration with University of Nottingham with 17 interaction designers and computer scientists participating in the workshop activities.

As part of the two-day workshop, we ran a session focused on exploring biosensing. Similar to work conducted in previous stages, we aimed at enabling participants to deepen their somatic awareness with the help of biodata, focusing on firstperson experiences, and potentially spurring creativity for use cases in the context of balance. Participants were divided into four groups focusing on different scenarios around balance, and were given different biosensing technologies. Similarly to the second stage, in each group, each person took turns in the role of the person wearing a sensor, while the others took the role of the observers. Building on the learnings from the previous stages, the person wearing the sensor was asked not to look at the screen depicting real-time biodata graphs. Instead, the others in the team were asked to watch the screen and to engage the wearer in balancing acts. In this way, the wearer could engage in feeling aspects of the balancing activity without being distracted by the graphical representation of biodata on a screen. As we explored alternatives to move away from screen-based representations of biodata, following learnings from the previous stage, we had designed a coupling that mapped muscle contractions as measured by EMG, to sound outputs. Because it was generating sound, the EMGsignal could simultaneously be experienced by all participants - including the wearer. This very simple interaction spurred the kinds of designerly explorations of sensing that we had been seeking.

\section{Stage 4: Couplings of Sensing and Actuation}

In our final stage, we focused on exploring the potential of crafting artefacts that couple sensing and actuation, enabling collective felt experiences of biosensing as this had been successful in the third stage. In collaboration with the Catholic University of Milan, we organised a design workshop with 16 participants from interdisciplinary backgrounds ranging from interaction design, clinical psychology, and computer science around the theme of "synchrony" (Figure 1c). Synchrony can be broadly defined as a spontaneous synchronisation of a range of neural, physiological and behavioural responses when people interact particularly through e.g. synchronised bodily movement [29]. As in previous stages, we followed a soma design methodology and included a session for biodata exploration. Participants were divided into four groups, each group exploring synchrony with different biosensors, e.g. through movement, breathing, emotional states, or mindfulness states.

Here we focus on two particular couplings that were designed in this stage and were brought to the workshop: 1) an artefact that couples EDA data from one participant with temperature change actuation felt by another participant, and 2) an artefact that couples acceleration data from two participants mapped to changes in a soundscape. Both were built specifically to expose biodata qualities in the context of synchrony.

\section{RESULTS: FROM BIODATA TO SOMADATA}

Throughout our design research process we explored coupling several different sensing and actuation modalities, until we found those that were most relevant to the design setting. Our explorations were guided by the soma design methods, helping to determine which ones we finally brought to the workshops. As we hinted above, the coupling of EMG to sound in the third stage of our work, and the couplings introduced in the fourth stage of our work, led to successful design processes. But what qualifies as 'successful'? To us, it was important that any design ideas arising in the workshops would be firmly grounded in, firstly, what the biodata technology affords and can realistically measure with some validity. Furthermore, that there would be a clear link between the experience of the somadata to a design idea. But perhaps more importantly, that the design ideas would be true 'discoveries' of novel experiences and interactions - not documented before - firmly grounded in the realities of our somatic experiences. Below we introduce three such discoveries that emerged when workshop participants experienced and played with somadata.

\section{Balance Premonition}

Electromyography (EMG) measures electrical currents generated in muscles during their contraction [37]. An EMG signal can be characterised as a rapidly oscillating signal with varying positive and negative amplitude, where the absolute value of the amplitude represents the amount of contraction in the muscle. Estimating muscle contractions by observing an EMG graph is difficult as the differences in amplitude can be quite subtle.

As mentioned above, during the third stage of our work we designed a coupling to expose how muscle contraction works and make it available for designers to work with. We will now detail some of our design decisions behind making this coupling. First, sound as an output can be used to represent subtle variations in biodata, as it relies on the high fidelity of the auditory human perception system [16]. We mapped the EMG signal to sound in three steps: first, we took the absolute value of the signal - a full-wave rectification; second, we smoothed it with a low-pass filter to remove some of the oscillation; finally, the smoothed signal was mapped to the pitch of a sine wave generator. The higher the measured muscle contraction the higher the pitch the generator produces, and vice versa (see Figure 2a).

At the workshop focused on balance (see Stage 3), where we brought this coupling, participants first tested the EMG 

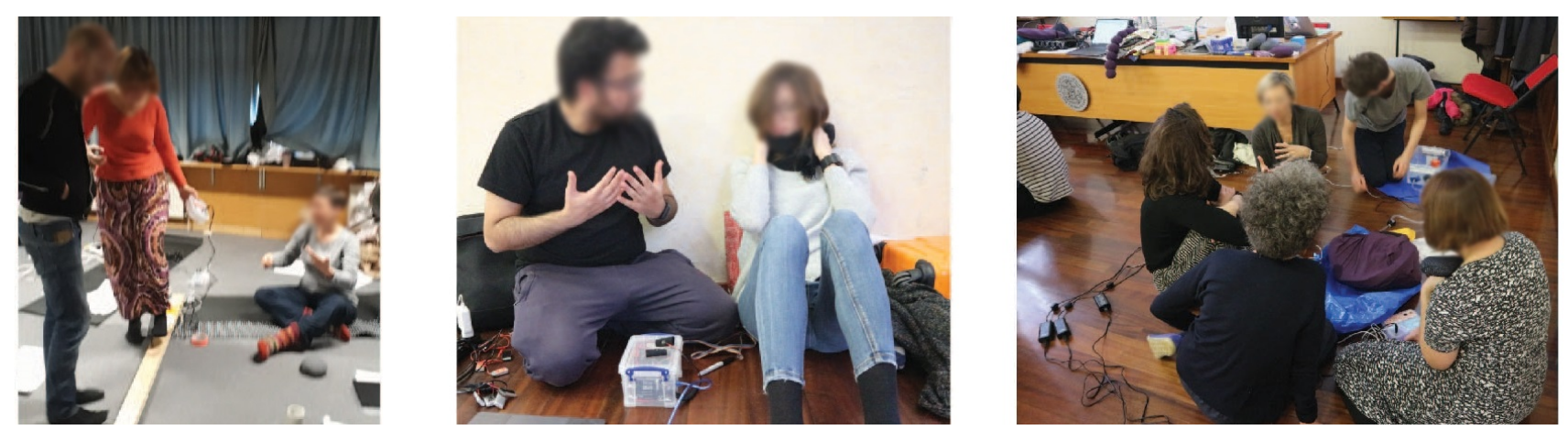

Figure 1. Images from our workshops where participants share and discuss their first-person experiences of trying the couplings (From left to right): a) Exploring the "Balance Premonition" coupling while balancing on the rod b) Experiencing the warm and cold sensations of the "Breeze Around Your Neck" coupling c)Group reflection after trying out Sync-inspired couplings

sensor when connected to a biodata visualisation software. The sensor consisted of three electrodes attached to a muscle on the participant's lower leg that acquired the EMG signal and represented it as a graph. With this setup we explored how several muscles in this area of the body would contract while performing activities like walking and balancing on a balancing rod (Figure 1a) emulating a tightrope. In the context of balance, ECG and ACC were also explored, but EMG was picked because of the accuracy in capturing muscles that we realised were involved in walking on a tightrope. Sound actuation, rather than e.g. vibration, was the one that offered the shared insight reported while capturing subtle variations and keeping a focus on the bodily activity.

The participant wearing the biosensor was balancing on the rod, while other workshop participants observed the screen showing the real-time generated graph of the registered signal. After testing this setup for a while, one particular muscle seemed to indicate when one was about to lose balance and fall off the rod. The ones watching the visualisations on the screen could sort of predict that moment and they would say loud: "And now you will soon fall off!", right as he was falling. As a follow-up exploration in this particular setting, we tested the EMG to sound coupling that we have had to the workshop. For the person balancing, the coupling between EMG biodata and sound was a central part of this dynamically unfolding balancing experience. As one participant put it: "Compared to the screen visualisations that were disconnecting the perception of my leg while balancing, since the data were viewed and interpreted by others for me, the audio output allowed me to perceive what the biosensor was measuring in my body, while also being able to focus on the balancing act.".

Additionally, the change in frequency made the nuances of the muscle contraction very apparent and allowed the whole team to share and experience the balancing act while watching and following the movements of the person trying to balance on the rod. Instead of dividing their attention between watching a screen and interpreting the movements of the balancer, there was a shift from the pure observation and output of discrete data to a shared first-person experience of balancing. As articulated in a first-person account from one of the participants: "While balancing on the rod, everybody could hear the changes of my contracting leg muscle. This changed the perception of my balancing act. I could focus much more on this muscle and link it to the balancing as I could experience its movement in real time".

The 'discovery' here is not only which muscle we could use to predict losing balance, but also how we could use sound in an interaction to let wearers get a different, more nuanced, experience of their own balance - right before falling off - a premonition of sorts. This experience of "knowing-beforefalling-off" is a new somaesthetic experience that involves a deepened understanding of your own body. This was a novel experience for us, which we could not have known before the workshop. The coupling itself did not make the person balancing to perform better. But it made them more aware on how the body is involved and participates in the act of balancing, and also going off-balance, as they could "hear" (and in turn "feel") the actual balancing act through their biodata in a tacit, less interpretive way compared to screen visualisations. Finally, the sound enabled a shared experience of one's first-person account of their own body, as the whole group could participate in the act of balancing and falling. The discovery around the Balance Premonition coupling became an important stepping-stone in our understanding and articulation of couplings as somadata.

\section{A Breeze Around Your Neck}

Electrodermal activity (EDA) is linked to physiological arousal resulting from activity in the sympathetic nervous system. It is often considered a difficult biosignal to work with [41], as changes responding to skin conductance can happen due to many different reasons, including strong positive emotion, strong negative emotion, as well as increased physical activity. Inspired by the body practices and sensitising activities used in our design process, we wanted to use actuation applied on the body. While designing for synchrony could be seen from the perspective of signals that we control, such as movement or breathing, synchronising EDA changes with feedback effects or nudges perceived on the body emerged as way to disrupt the user's role in EDA interactions.

Following up on our previous work on material representations of EDA mapped to thermochromic displays [58, 59], we found 


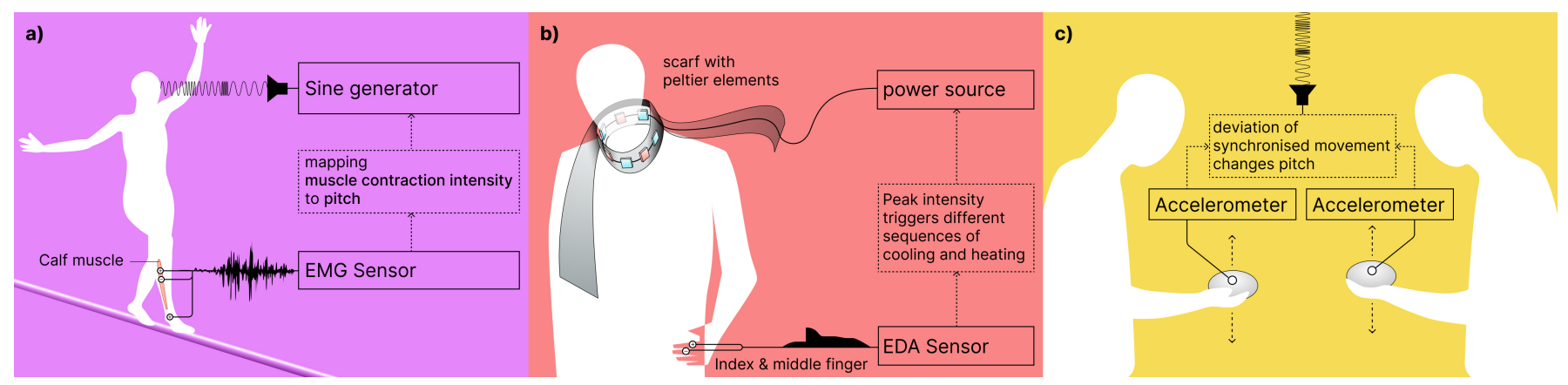

Figure 2. Diagrams of the three somadata couplings. (From left to right): a) "Balance Premonition" couples electromyography (EMG) biodata with sonic output, b) "A Breeze Around Your Neck" couples electrodermal activity (EDA) biodata with temperature, and 3) "Sounds of Synchronous Movements" couples acceleration data (ACC) with sound.

that mapping EDA to haptic output, and particularly heat, can create evocative sensations on the skin. Heat is known to be quite an intimate modality [27], which has been used to relieve pain, relax muscles, or to raise or lower the overall body temperature [55]. To explore the combination of EDA and heat we designed a coupling that mapped the rapidly occurring fluctuations, known as skin conductance responses (SCR), to heat feedback. We integrated four 20 × $20 \mathrm{~mm}$ Peltier elements in a woollen scarf, which was connected to the SCR signal. Peltier elements are small modules explored previously for providing temperature feedback $[64,59]$. They were driven by an Arduino and were actuated by the SCR signal, using a filter to smooth out the noise present in the signal. With a sampling frequency of 20 samples/second, we collected five seconds worth of SCR data and fed it into a peak detection algorithm. Depending on the size of the peak, one, two, three or four Peltier elements would turn on. The higher the peak, the more elements would turn on, one by one over time. Through this mechanism, a sensation of heat moving from one place to the next could be achieved, representing an increasing arousal level.

The reason for choosing this mapping was to communicate to the wearer the level or intensity of arousal, as existing research highlights the importance of biosensory interfaces to express multiple levels of affective states, supporting people to understand and interpret their biodata [59]. At the workshop, where we brought this coupling (see Stage 4 above), when wearing and experiencing the scarf (Figures 1b, 2b), we found that it was hard to detect a wave of heat going around the neck. This was because the material of the scarf (wool) was too warm to allow for perceiving subtle and moving temperature changes. However, Peltier elements are interesting in that they get warm on one side, but on the other, they simultaneously get cold. Thus, participants started experimenting with turning the elements around to get a cold wave. The cold sensation was perceived by the wearers much more clearly and was found to be more pleasant than the warm one.

While experiencing the properties of heat and cold in relation to the registered SCR biodata, we asked ourselves: "What if cold patterns could simultaneously convey the decay of an EDA signal and a perceivable pleasant temperature dissipating on the skin?". By discussing the felt experience of biodata, in the form of heat (and cold)-based actuation, participants used the cold sensation to further explore and challenge the data registered from the EDA. Through this coupling we reflected on the fact that SCR are not only about how the signal rises to show arousal levels but also how it fades out and how we can relate it to the dynamics of heat dissipation. That insight finally allowed us to think how we could in turn appropriate heat changes not only to show arousal but also to create interactions that can soothe users.

Based on this understanding, participants tried turning only every second Peltier element around, creating a combination of cold and warm areas that could be felt on the skin of the wearer. Triggered by the SCR signal, some Peltier elements would warm up the fabric and others would yield a cooling pattern in order to create a distinct, discernible and somewhat "pointed" experience of a cooling sensation. Although the sensation of temperature is generally subjective, everyone who tried the scarf found the cold sensation to be pleasant. As described by participants: "the cold effect feels mild and pleasant", "it feels like it's a breeze and it soothes”, “... kind of stroking you". The sensation of the pleasant "breeze" around the neck, instead of being a cold wave, was a distinct movement of temperature changes felt around the neck, and created by the person's arousal levels.

Our 'discovery' here emerged from the observation that it was difficult to communicate consecutive arousal peaks with temperature, as time is required to spread the changes from previous arousal levels. However, once participants were sensitised, they appropriated the coupling and tweaked it to make us realise that heat and cold sensations could be combined in order to convey a felt and aesthetically evocative experience of arousal biodata dissipating, opening up the design space of designing with EDA in novel and unexpected ways.

\section{Sounds of Synchronous Movements}

An accelerometer (ACC) is a device capable of tracking how speed evolves in a given direction axis. Uniaxial ACCs are able to detect acceleration in one direction (e.g. only vertically, laterally, or forward and backwards). For the workshop focused on the topic of synchrony (see Stage 4 above), we designed a coupling in a form of two artefacts (Figure 3c) that were meant to be hand-held by different people, each 
hosting a uniaxial ACC. They were linked to the BITalino [7, 5] biosignal platform and a simple algorithm that processed and mapped acceleration differences between the two ACC artefacts to pitch modulation. The coupling, inspired by the topic of synchrony, was made to show differences in movement between the two artefacts. The acceleration differences between the measured movements were mapped to a MIDI sound generation system, creating a chord with a pitch modified in accordance to the level of synchronisation between movements.

For this particular case of designing for synchrony, we were interested in exploring movement. After testing EMG, the use of accelerometry turned out to work better in a context with much more freedom than a particular balance task. The fact that no specific muscle constraints nor electrodes were needed suggested sound and movement could be explored afresh. During the designing of this coupling we were inspired by the range of possibilities that audio feedback could offer, such as changes in harmony, speed, volume or pitch, and how those could be mapped to performed movements. Similar to the EMG coupling described earlier, we chose pitch, since we found it best for representing perceptible small signal changes.

During the workshop, participants freely explored choreographies through synchronised and unsynchronised movements, sensitising themselves to the possibilities and limitations of using ACC data through the coupling. Some participants were able to carry out a joint, synchronous experience, exploring communication - sometimes without keeping eye-contact or talking at all, only driven by the felt experience of synchronising their movements that were in turn translated into sound. Some participants even felt prompted to challenge the coupling vis-à-vis their felt experience and creatively "hacked" the artefacts, creating novel somaesthetic experiences that felt most evocative, playful, or meaningful to them. These hacks are important as they created design opportunities that we did not foresee before the workshop.

Among other materials we brought to the workshop, there was a collection of soft three-dimensional shapes made of foam and textile, equipped with pockets for sensors or actuators to be placed inside the shapes and experienced at different parts of the body [65]. Two participants picked one such shape and inserted the ACC biosensor inside. The combination of the sound coupling and the soft textile shape allowed them to play with different weights and placements of the biosensor on the body - limbs, on top of the head, or even as a tail, resonating with previous HCI design work [54]. They found that different shapes and placements on the body completely changed their synchronous experience, embracing the sensor as an extension of the body. As part of this exploratory and playful context of experiencing the coupling, an interesting design discovery emerged. The interaction experienced through sound, which changed according to the movements that participants creatively performed together, mediated non-verbal body communication that defied the topic of the workshop, and made participants think beyond synchrony. As articulated by one of the participant designers: "The audio actuation that was meant to hint and hold two participants together, evolves to be the means of communication between them, mediating a non-verbal dialogue that leads to choreographies".

The audio feedback was first successful in revealing synchronybased interactions that foregrounded how bodies move in relation to others, as opposed to visualisation on a display that requires eye contact and thus can compromise moving freely in space. But the 'discovery' here was that the coupling of acceleration differences to sound modification successfully engaged participants in sharing and communicating movements in a way we could not have thought of, leading them to the creation of novel ways of moving together. The somadata coupling took us out of the "habitual" way of looking at ACC data and the original design context of synchrony, prompting a shared multiuser understanding beyond the attention on a single participant's soma movement.

\section{DISCUSSION}

As we have shown, turning biodata into a 'material' - into somadata - allowed designers to come up with somaticallygrounded design discoveries. But coming back to the complexities of what sensors really measure and how that in turn relates to our somatic, often quite complex, realities, we might wonder what the possibilities and limitations of this approach are? We know that, in general, the ways that data is collected, aggregated, and presented always enables certain possibilities for action and constrains others, making some things visible and others invisible. For example, standardised representations based on graphs and timelines privilege comparison and standardisation, useful for scientific analysis. Alternative representations could encourage reflection on the cyclical passage of time [60], encourage sensemaking through hand-drawing [49], or encourage storytelling [17].

The workshops conducted were grounded on soma design, including a combination of: bodily activities, sensitising techniques, exploration of digital materials. The last two workshops, focused on Balance and Synchrony, also included design work in the form of developing new interactive experiences for each context. Design work also took place before each workshop as the couplings were crafted for each context. All workshops reflect the path we took for engaging with and exploring biodata creatively. Sensitising and becoming attuned to materials is an important part of a design process for shaping materials and technologies into prototypes: In this paper we show that sensitising encounters with biosensors can be enriched, if the encounter between designers and biosensing technologies is scaffolded in ways that enable intimate reflections and allow for co-experiencing and sharing. This scaffolding should also take into account what we are designing for. For example, when designing for balance, it made sense to explore even the tiniest of muscle contractions, coupled with an output modality able to show those subtle variations. We also want to point out that a coupling of biodata to some specific modality might serve a generative role in the workshop - as a vehicle for meaning-making and discovery, but then, the final design might not necessarily build on that coupling directly. With somadata, rather than prescribing a particular way of collecting, aggregating and presenting data, we point at specific aspects that we found important when making 
biodata available as a material that can firstly be experienced, and then used to design with.

First, somadata is designed to spur first-person, felt, explorations. Similar to previous research in the domain of biosensing-enhanced emotional technologies [24, 58], an ongoing challenge for our explorations was how biosensing can be translated into and experienced through more than one of our senses. We wanted to experience biosensing in a tangible and somatic sense, instead of having to interpret it through an external, distant representation such as through a screen graph. By carefully coupling biodata to haptic and sonic actuators, we expose properties of muscle contractions, skin conductance and synchronised movement and make these available to be felt and played with. For example, with the somadata coupling presented in the "Balance premonition" section, the nuances of muscle contractions when walking on a balancing rod can be experienced while the balancer wholly concentrates on balancing, gaining a new understanding of how to balance and how EMG could be used to enrich the experience.

Second, somadata emphasises collaboration and participation, allowing for the emergence of interactions grounded on group design sessions. Making biodata available as a design material goes beyond a single first-person somatic experience towards shared first-person experiences. The act of designing somadata artefacts is also helpful for articulating and communicating to one another how the very particular properties of digital materials can be exposed to surface their unique qualities. This is in line with what Höök [20] refers to as the sociodigital material for designing interactive technologies. By sociodigital materials she refers to the combination of the digital materials and our social and living bodies that we design with and for also changing in and through the interactions we perform. For example, in a somadata coupling that translates EDA patterns into heat felt on the skin, like the one we presented here, the inherent time dynamics in the chosen heating material open the possibility of playing with gradual heating sensations travelling on the skin, making the electrodermal information into something soft and sensuous. And ultimately also potentially making us more aware of our arousal - its shape and form perhaps even altering the way we react. Such a perspective of designing with sensed and actuated data in relation to bodies and sociodigital materials is what the concept somadata communicates.

Finally, we are not claiming that our approach will work for every design process involving biosensors. Couplings are particular ways of representing biodata, narrowed down to specific settings and showing specific features of biodata. By designing couplings in one way, we are precluding many other possible ways of engaging with biodata. We are also not claiming that every feature of every type of biosensor would be possible to represent in a tangible, felt way. Some biodata features may represent very long-term trends that would challenge the real-time experimentation with biodata that we privileged in our process in the soma design workshops. Additionally, some types of biodata may be very intimate $[19,51]$ and require special care when sharing within a design team. Furthermore, we limited ourselves to one-to-one couplings.
A future step would be to explore multiple sensory inputs to multiple actuation outputs. We discuss some of this future work below.

\section{Making (Soma)data Available to Design}

Looking back at the overall approach we followed for exploring this research space, we note two main components. The first one is the importance of sensitisation methods, aimed at increasing one's ability to feel and articulate different bodily sensations and properties of materials. Sensitising methods were present in each workshop and research exploration, through body scans, Feldenkrais or A-Lab sessions. A second component of our approach was that, before each workshop, a substantial amount of preparations took place, focused on the respective workshop topic, and design explorations of biodata qualities and possibilities of actuation. Preparing somadata couplings was important for exploring and exposing biodata properties, which would then be brought to the workshop to be further explored by the workshop participants.

Data from biosensors, rather than being "raw", becomes in fact a carefully crafted sociodigital material, readily to be experienced and molded by workshop participants. This of course requires that we are able to prepare the most relevant somadata for a particular design context. As in any design process, the materials provided will delimit the design space at the same time as it is exactly in this delimitation that creativity can flow. Reflecting on future directions of making somadata couplings more available and malleable to design with, it would be important to have some type of platform that would allow designers to change and adapt them "on the fly", based on new discoveries that might happen during a workshop or a design process. In an example from our own ongoing work, we are currently exploring the potential of EMG as a design material for opera singers, allowing them to observe how different muscles involved in singing, as well as breathing while singing, can be used as input for designing new types of multimedia performances. For this setting, it would be desirable, from the designer's perspective, to have direct access to ways of shaping and coupling not only EMG but also EDA and ACC data to different types of output modalities such as sounds, light or shape-changing materials. Such a platform could include the integration of visual programming interfaces, providing the possibility to run signal processing code snippets to assist the design of couplings. In shaping somadata and when sensitising ourselves to the qualities of each coupling, we note that it is important to shift the focus away from the constraints posed by technology, and instead highlight possibilities revealed by each coupling and how can they contribute to the crafting of novel somaesthetic experiences.

\section{From Couplings to Crafting Interactive Experiences}

The exploration of biosensing in the form of couplings between biodata and actuation on the body encouraged reflections with regard to different paths for designing orchestrated and elaborately crafted interactive experiences. In order to properly expose and experiment with what the dynamic unfoldings of the coupled biodata might bring over time, we need to develop a way to dynamically orchestrate the interactions with a particular coupling over time. The term orchestration 
here refers to the process of crafting the links with the different mappings between a chosen sensor and a chosen actuator, for registering and translating a particular event or movement.

Therefore, apart from exposing the material properties of biosensors in order to explore and design with these, somadata couplings also suggest paths towards possible final designs. Couplings should not be considered as quick mappings between a chosen input and output modality. To let us, designers, imagine how they might be used in a final design, the mappings need to be carefully chosen to fit with the aims of the design and the qualities of biodata, as well as letting us imagine how, for example, long-term engagement might evolve and develop, beyond the first encounter. Our successful couplings are the result of deliberate choices vis-à-vis the aim of the design workshop where they were brought, alongside training our somaesthetic appreciation, our attention to the body senses, and our willingness to engage with the biosensory material. But at the same time, the examples of somadata presented here would need further development and fine tuning of their orchestration between sensing and actuating in order to render a truly somaesthetic experience, for a specific design context.

Additionally, we observed that having sensing-actuation couplings can expand the possibilities of designing for interesting and evocative somaesthetic experiences, to also explore the potential of designing evocative and engaging interactions with the technology itself. For example, in thinking how to design the coupling that maps EDA to heat experienced on the neck, and how the orchestration would evolve over time, we realised that actuation sequences extend the perception of the intended body characteristics. The inherent time dynamics in the chosen heating material trigger our imagination to a spectrum of interactions that expand to an implicit level, beyond a one-to-one mapping, and which would be welcomed. Such implicit interactions enabled through this coupling could, for example, exploit the unexplored potential of our skin to sense thermal gradients, and turn information inputs sensuous.

\section{From First-person Accounts to Shared Experiences}

Design is often a collaborative activity. During our workshops which unfolded in collaborative design settings, we experienced that, to this "social" aspect the couplings can firstly contribute with creating empathy and understanding for other bodies, beyond your own. This can lead to further understanding and appreciating your own body as well-its limitations and possibilities. By empathy we do not mean to empathise with vulnerable user groups by engaging in empathising activities as part of a research process, which has been recently critiqued by Bennett and Rosner [6]. On the contrary, we agree with their perspective, and assert that empathy for others can arise by engaging with other bodies actively, instead of observing or studying those from a distance, building on their idea of creating partnerships. Couplings of sensing and actuation can be used for exploring this path towards empathy grounded in and through one's actual body and somadata, which is something that we have already started to explore and study further in the context of depression and anxiety [1]. In our soma design workshops we purposely engaged in group design exercises and bodily activities, bringing together several first-person voices to compensate for the single first-person limitations already noted in [21]. Somaesthetics and soma design already acknowledge that bodies are different, and diversity must be embraced to give a truthful account of what a design result is [20]. And this is one reason why soma design proved to be a suitable method to explore biosensing, as it opened our design space to account for a more holistic understanding of sensing, in addition to making sense of bodily data. In future work, we would like to explore engagements with somadata also from a feminist perspective [26], building on our previous work on the politics of the body [22].

Additionally, we believe that the component of shared experiences enabled by somadata couplings emerges in the presence of certain technology links, such as coupling synchronous movements through ACC biosensors to a soundscape, and that these are key in discovering new shared experiences. Although our examples were built upon simple interactions between few people, they inspired us to further research the potential of shared experiences, mediated through couplings. The explorations we conducted and the couplings we developed and tested provided a fruitful ground to probe what sharing adds to the somadata. With a coupling having a concurrent impact on more than a single person, one can gain a broader perspective on how collaborative and shared interactions can be crafted when leveraging biodata as somadata. Specifically, how biodata can make sense and be experienced in a tangible form not only by a single person, or by the person whose data are registered through a sensor, but also in contexts of communicating and sharing experiences, as for example, one person feeling another person's arousal as a cooling pattern on their neck.

\section{CONCLUSION}

Through a range of soma design-inspired workshops we explored how to surface interesting qualities in biodata in order to spur designerly imaginations. The most fruitful explorations came from engaging with what we frame as somadata - that is turning the biodata into a 'tangible' form by coupling it to sensuous actuation, enabling first-person, felt encounters. We also found that once the somadata became present in the room, attainable for exploration, collaborative meaning-making processes around experiencing and designing with biodata arose. We presented three somadata materials: 1) electromyography turned into sound, 2) electrodermal activity turned into temperature and 3) acceleration data turned into soundscape modulation. All three led to interesting design discoveries firmly grounded in the realities of our somatic experiences, through supporting shared experiences and meaning-making processes in the design team and, through showing us a path towards crafting interactive somaesthetic experiences with technologies that take into account our whole somas.

\section{ACKNOWLEDGMENTS}

This work has been supported by AffecTech: Personal Technologies for Affective Health, Innovative Training Network funded by the H2020 People Programme under Marie Skłodowska Curie grant agreement No 722022, the Swedish Foundation for Strategic Research project RIT15-0046, and the Swedish Research council project 2016-04709. 


\section{REFERENCES}

[1] AffecTech. 2017. Personal Technologies for Affective Health. (Jan 2017). Retrieved Sep 10, 2019 from https://www. affectech.org/

[2] Cheryl Akner-Koler and Parivash Ranjbar. 2016. Integrating Sensitizing Labs in an Educational Design Process for Haptic Interaction. FormAkademisk forskningstidsskrift for design og designdidaktikk 9, 2 (Aug. 2016). DOI :

http://dx.doi.org/10.7577/formakademisk. 1269

[3] Miquel Alfaras, Miguel Cornelles Soriano, and Silvia Ortín. 2019. A fast machine learning model for ECG-based heartbeat classification and arrhythmia detection. Frontiers in Physics 7 (2019), 103. DOI: http://dx.doi.org/10.3389/fphy. 2019.00103

[4] Kristina Andersen and Ron Wakkary. 2019. The Magic Machine Workshops: Making Personal Design Knowledge. In Proceedings of the 2019 CHI Conference on Human Factors in Computing Systems (CHI'19). ACM, New York, NY, USA, Article 112, 13 pages. DOI : http://dx.doi.org/10.1145/3290605.3300342

[5] Diana Batista, Hugo Plácido da Silva, Ana Fred, Carlos Moreira, Margarida Reis, and Hugo Alexandre Ferreira. 2019. Benchmarking of the BITalino biomedical toolkit against an established gold standard. Healthcare Technology Letters 6, 2 (April 2019), 32-36. DOI : http://dx.doi.org/10.1049/htl.2018.5037

[6] Cynthia L. Bennett and Daniela K. Rosner. 2019. The Promise of Empathy: Design, Disability, and Knowing the "Other". In Proceedings of the 2019 CHI Conference on Human Factors in Computing Systems (CHI'19). ACM, New York, NY, USA, Article 298, 13 pages. DOI : http://dx.doi.org/10.1145/3290605.3300528

[7] BITalino 2013. BITalino. (2013). Retrieved Sep 10, 2019 from http://www. bitalino.com

[8] Kirsten Boehner, Phoebe Sengers, and Simeon Warner. 2008. Interfaces with the Ineffable: Meeting Aesthetic Experience on Its Own Terms. ACM Trans. Comput.-Hum. Interact. 15, 3, Article 12 (Dec. 2008), 29 pages. DOI :

http://dx.doi.org/10.1145/1453152.1453155

[9] Magnus Boman and Pedro Sanches. 2015. Sensemaking in Intelligent Health Data Analytics. KI - Künstliche Intelligenz 29, 2 (Jan. 2015), 143-152. DOI : http://dx.doi .org/10.1007/s13218-015-0349-0

[10] Yekta Said Can, Bert Arnrich, and Cem Ersoy. 2019. Stress detection in daily life scenarios using smart phones and wearable sensors: A survey. Journal of Biomedical Informatics 92 (April 2019), 103139. DOI : http://dx.doi.org/10.1016/j.jbi.2019.103139

[11] Nigel Cross. 2017. Design ability. NA 5, 4 (2017). http://arki tekturforskning.net/na/article/view/900
[12] Audrey Desjardins and Timea Tihanyi. 2019. ListeningCups: A Case of Data Tactility and Data Stories. In Proceedings of the 2019 on Designing Interactive Systems Conference (DIS '19). ACM, New York, NY, USA, 147-160. DOI : http://dx.doi.org/10.1145/3322276. 3323694

[13] Ludvig Elblaus, Vasiliki Tsaknaki, Vincent Lewandowski, and Roberto Bresin. 2015. Nebula: An Interactive Garment Designed for Functional Aesthetics. In Proceedings of the 33rd Annual ACM Conference Extended Abstracts on Human Factors in Computing Systems (CHI EA '15). ACM, New York, NY, USA, 275-278. DOI :

http://dx.doi.org/10.1145/2702613.2725454

[14] Sarah Fdili Alaoui. 2019. Making an Interactive Dance Piece: Tensions in Integrating Technology in Art. In Proceedings of the 2019 on Designing Interactive Systems Conference (DIS '19). ACM, New York, NY, USA, 1195-1208. DOI :

http://dx.doi.org/10.1145/3322276.3322289

[15] Ylva Fernaeus and Petra Sundström. 2012. The Material Move How Materials Matter in Interaction Design Research. In Proceedings of the Designing Interactive Systems Conference (DIS '12). ACM, New York, NY, USA, 486-495. DOI : http://dx.doi.org/10.1145/2317956.2318029

[16] E. Bruce Goldstein and James R. Brockmole. 2017. Sensation and perception (10th ed.). Cengage Learning, Boston. OCLC: 952665360.

[17] Dize Hilviu and Amon Rapp. 2015. Narrating the Quantified Self. In Adjunct Proceedings of the 2015 ACM International Joint Conference on Pervasive and Ubiquitous Computing and Proceedings of the 2015 ACM International Symposium on Wearable Computers (UbiComp/ISWC'15 Adjunct). ACM, New York, NY, USA, 1051-1056. DOI: http://dx.doi.org/10.1145/2800835.2800959

[18] Victoria Hollis, Artie Konrad, Aaron Springer, Matthew Antoun, Christopher Antoun, Rob Martin, and Steve Whittaker. 2017. What Does All This Data Mean for My Future Mood? Actionable Analytics and Targeted Reflection for Emotional Well-Being. Hum.-Comput. Interact. 32, 5-6 (Nov. 2017), 208-267. DOI : http://dx.doi.org/10.1080/07370024.2016.1277724

[19] Sarah Homewood. 2018. Designing for the Changing Body: A Feminist Exploration of Self-Tracking Technologies. In Extended Abstracts of the 2018 CHI Conference on Human Factors in Computing Systems (CHI EA '18). ACM, New York, NY, USA, Article Paper DC11, 4 pages. DOI :

http://dx.doi.org/10.1145/3170427.3173031

[20] Kristina Höök. 2018. Designing with the Body: Somaesthetic Interaction Design (Design Thinking, Design Theory). The MIT Press, Cambridge, MA. 
[21] Kristina Höök, Baptiste Caramiaux, Cumhur Erkut, Jodi Forlizzi, Nassrin Hajinejad, Michael Haller, Caroline Hummels, Katherine Isbister, Martin Jonsson, George Khut, Lian Loke, Danielle Lottridge, Patrizia Marti, Edward Melcer, Florian Müller, Marianne Petersen, Thecla Schiphorst, Elena Segura, Anna Ståhl, Dag Svanæs, Jakob Tholander, and Helena Tobiasson. 2018. Embracing First-Person Perspectives in Soma-Based Design. Informatics 5, 1 (Feb. 2018), 8. DOI : http://dx.doi.org/10.3390/informatics5010008

[22] Kristina Höök, Sara Eriksson, Marie Louise Juul Søndergaard, Marianela Ciolfi Felice, Nadia Campo Woytuk, Ozgun Kilic Afsar, Vasiliki Tsaknaki, and Anna Ståhl. 2019. Soma Design and Politics of the Body. In Proceedings of the Halfway to the Future Symposium 2019 (HTTF 2019). Association for Computing Machinery, New York, NY, USA, Article Article 1, 8 pages. DOI :

http://dx.doi.org/10.1145/3363384.3363385

[23] Kristina Höök, Caroline Hummels, Katherine Isbister, Patrizia Marti, Elena Márquez Segura, Martin Jonsson, Florian "Floyd" Mueller, Pedro A.N. Sanches, Thecla Schiphorst, Anna Ståhl, and et al. 2017. Soma-Based Design Theory. In Proceedings of the 2017 CHI Conference Extended Abstracts on Human Factors in Computing Systems (CHI EA '17). ACM, New York, NY, USA, 550-557. DOI :

http://dx.doi.org/10.1145/3027063.3027082

[24] Noura Howell, John Chuang, Abigail De Kosnik, Greg Niemeyer, and Kimiko Ryokai. 2018. Emotional Biosensing. Proceedings of the ACM on Human-Computer Interaction 2, CSCW (Nov 2018), 1-25. DOI : http://dx.doi .org/10.1145/3274338

[25] Noura Howell, Laura Devendorf, Rundong (Kevin) Tian, Tomás Vega Galvez, Nan-Wei Gong, Ivan Poupyrev, Eric Paulos, and Kimiko Ryokai. 2016. Biosignals As Social Cues: Ambiguity and Emotional Interpretation in Social Displays of Skin Conductance. In Proceedings of the 2016 ACM Conference on Designing Interactive Systems (DIS '16). ACM, New York, NY, USA, 865-870. DOI :

http://dx.doi.org/10.1145/2901790.2901850

[26] Nassim JafariNaimi and Anne Pollock. 2018. Heart Sense: experiments in design as a catalyst for feminist reflections on embodiment. In DRS2018: Catalyst. Design Research Society. DOI :

http://dx.doi.org/10.21606/drs.2018.409

[27] Martin Jonsson, Anna Ståhl, Johanna Mercurio, Anna Karlsson, Naveen Ramani, and Kristina Höök. 2016. The Aesthetics of Heat: Guiding Awareness with Thermal Stimuli. In Proceedings of the TEI '16: Tenth International Conference on Tangible, Embedded, and Embodied Interaction (TEI '16). ACM, New York, NY, USA, 109-117. DOI :

http://dx.doi .org/10.1145/2839462.2839487
[28] George (Poonkhin) Khut. 2006. Development and Evaluation of Participant-Centred Biofeedback Artworks. Ph.D. Dissertation. School of Communication Arts, University of Western Sydney. http://www.georgekhut.com/doctoral-exegesis/

[29] Sander L. Koole and Wolfgang Tschacher. 2016. Synchrony in psychotherapy: A review and an integrative framework for the therapeutic alliance. Frontiers in Psychology 7, June (2016), 1-17. D0I : http://dx.doi.org/10.3389/fpsyg. 2016.00862

[30] Wonjun Lee, Youn-kyung Lim, and Richard Shusterman. 2014. Practicing Somaesthetics: Exploring Its Impact on Interactive Product Design Ideation. In Proceedings of the 2014 Conference on Designing Interactive Systems (DIS '14). ACM, New York, NY, USA, 1055-1064. DOI : http://dx.doi.org/10.1145/2598510.2598561

[31] Lian Loke and Toni Robertson. 2013. Moving and Making Strange: An Embodied Approach to Movement-based Interaction Design. ACM Trans. Comput.-Hum. Interact. 20, 1, Article 7 (April 2013), 25 pages. DOI : http://dx. doi.org/10.1145/2442106.2442113

[32] Andrés Lucero, Audrey Desjardins, Carman Neustaedter, Kristina Höök, Marc Hassenzahl, and Marta E. Cecchinato. 2019. A Sample of One: First-Person Research Methods in HCI. In Companion Publication of the 2019 on Designing Interactive Systems Conference 2019 Companion (DIS '19 Companion). ACM, New York, NY, USA, 385-388. DOI : http://dx.doi .org/10.1145/3301019.3319996

[33] Helena M. Mentis, Jarmo Laaksolahti, and Kristina Höök. 2014. My Self and You: Tension in Bodily Sharing of Experience. ACM Trans. Comput.-Hum. Interact. 21, 4 (June 2014), 20:1-20:26. DOI : http://dx. doi .org/10.1145/2617945

[34] Maurice Merleau-Ponty. 2002. Phenomenology of Perception. Routledge, London, UK.

[35] Carman Neustaedter and Phoebe Sengers. 2012. Autobiographical Design: What You Can Learn from Designing for Yourself. Interactions 19, 6 (Nov. 2012), 28-33. DOI : http://dx.doi .org/10 . 1145/2377783.2377791

[36] Catherine Pelachaud. 2012. Emotion-oriented systems. ISTE, London. OCLC: 992847532.

[37] M.B.I. Raez, M.S. Hussain, and F. Mohd-Yasin. 2006. Techniques of EMG signal analysis: detection, processing, classification and applications. Biological Procedures Online 8 (March 2006), 11-35. DOI: http://dx.doi.org/10.1251/bpo115

[38] Johan Redström. 2005. On Technology as Material in Design. Design Philosophy Papers 3, 2 (2005), 39-54. DOI : http://dx.doi.org/10.2752/144871305X13966254124275 
[39] John Rooksby, Mattias Rost, Alistair Morrison, and Matthew Chalmers. 2014. Personal Tracking As Lived Informatics. In Proceedings of the SIGCHI Conference on Human Factors in Computing Systems (CHI '14). ACM, New York, NY, USA, 1163-1172. DOI : http://dx. doi .org/10.1145/2556288.2557039

[40] Pedro Sanches, Kristina Höök, Corina Sas, and Anna Ståhl. 2019. Ambiguity As a Resource to Inform Proto-Practices: The Case of Skin Conductance. ACM Trans. Comput.-Hum. Interact. 26, 4, Article 21 (July 2019), 32 pages. DOI :

http://dx.doi.org/10.1145/3318143

[41] Pedro Sanches, Kristina Höök, Elsa Vaara, Claus Weymann, Markus Bylund, Pedro Ferreira, Nathalie Peira, and Marie Sjölinder. 2010. Mind the Body!: Designing a Mobile Stress Management Application Encouraging Personal Reflection. In Proceedings of the 8th ACM Conference on Designing Interactive Systems (DIS '10). ACM, New York, NY, USA, 47-56. DOI : http://dx . doi .org/10.1145/1858171.1858182

[42] Pedro Sanches, Axel Janson, Pavel Karpashevich, Camille Nadal, Chengcheng Qu, Claudia Daudén Roquet, Muhammad Umair, Charles Windlin, Gavin Doherty, Kristina Höök, and Corina Sas. 2019. HCI and Affective Health: Taking Stock of a Decade of Studies and Charting Future Research Directions. In Proceedings of the 2019 CHI Conference on Human Factors in Computing Systems (CHI '19). ACM, New York, NY, USA, Article 245, 17 pages. DOI: http://dx.doi.org/10.1145/3290605.3300475

[43] Corina Sas and Zhang Chenyan. 2010a. Do emotions matter in creative design?. In Proceedings of the 8th ACM Conference on Designing Interactive Systems (DIS'10). ACM, New York, NY, USA, 372-375. DOI : http://dx.doi .org/10.1145/1858171.1858241

[44] Corina Sas and Zhang Chenyan. 2010b. Investigating emotions in creative design. In Proceedings of the 1st DESIRE Network Conference on Creativity and Innovation in Design (DESIRE'10). ACM, New York, NY, USA, 138-149.

http://dl. acm. org/citation. cfm?id=1854969. 1854990

[45] Corina Sas and Carman Neustaedter. 2017. Exploring DIY Practices of Complex Home Technologies. ACM Trans. Comput.-Hum. Interact. 24, 2, Article 16 (April 2017), 29 pages. DOI :

http://dx.doi.org/10.1145/3057863

[46] seeed. 2019. Grove - GSR Sensor. (2019). http://wiki. seeedstudio.com/Grove-GSR_Sensor/

[47] John L. Semmlow. 2014. Biosignal and Medical Image Processing. CRC Press.

[48] Hugo Plácido Da Silva, Stephen Fairclough, Andreas Holzinger, Robert Jacob, and Desney Tan. 2015. Introduction to the Special Issue on Physiological Computing for Human-Computer Interaction. ACM Trans. Comput.-Hum. Interact. 21, 6, Article 29 (Jan.
2015), 4 pages. DOI: $\mathrm{http}: / / \mathrm{dx}$.doi .org/10.1145/2688203

[49] Jaime Snyder, Elizabeth Murnane, Caitie Lustig, and Stephen Voida. 2019. Visually Encoding the Lived Experience of Bipolar Disorder. In Proceedings of the 2019 CHI Conference on Human Factors in Computing Systems (CHI '19). Association for Computing Machinery, New York, NY, USA, Article Paper 133, 14 pages. DOI : http://dx.doi .org/10.1145/3290605.3300363

[50] Jordi Solsona Belenguer, Marcus Lundén, Jarmo Laaksolhati, and Petra Sundström. 2012. Immaterial Materials: Designing with Radio. In Proceedings of the Sixth International Conference on Tangible, Embedded and Embodied Interaction (TEI '12). ACM, New York, NY, USA, 205-212. DOI :

http://dx.doi.org/10.1145/2148131.2148177

[51] Marie Louise Juul Søndergaard and Lone Koefoed Hansen. 2016. PeriodShare: A Bloody Design Fiction. In Proceedings of the 9th Nordic Conference on Human-Computer Interaction. ACM, 113.

[52] Dag Svanæs. 1997. Kinaesthetic thinking: The tacit dimension of interaction design. Computers in Human Behavior 13, 4 (Dec. 1997), 443-463. DOI : http://dx. doi . org/10.1016/s0747-5632(97)00020-4

[53] Dag Svanæs. 2013. Interaction Design for and with the Lived Body: Some Implications of Merleau-ponty's Phenomenology. ACM Trans. Comput.-Hum. Interact. 20, 1, Article 8 (April 2013), 30 pages. DOI : http://dx.doi.org/10.1145/2442106.2442114

[54] Dag Svanæs and Martin Solheim. 2016. Wag Your Tail and Flap Your Ears: The Kinesthetic User Experience of Extending Your Body. In Proceedings of the 2016 CHI Conference Extended Abstracts on Human Factors in Computing Systems (CHI EA '16). ACM, New York, NY, USA, 3778-3779. DOI :

http://dx.doi .org/10.1145/2851581.2890268

[55] Perry S Tepperman and Michael Devlin. 1986. The therapeutic use of local heat and cold. Canadian Family Physician 32 (1986), 1110.

[56] Vasiliki Tsaknaki, Madeline Balaam, Anna Ståhl, Pedro Sanches, Charles Windlin, Pavel Karpashevich, and Kristina Höök. 2019. Teaching Soma Design. In Proceedings of the 2019 on Designing Interactive Systems Conference (DIS '19). ACM, New York, NY, USA, 1237-1249. DOI :

http://dx.doi .org/10.1145/3322276.3322327

[57] Panagiotis Tsiamyrtzis, Malcolm Dcosta, Dvijesh Shastri, Eswar Prasad, and Ioannis Pavlidis. 2016. Delineating the Operational Envelope of Mobile and Conventional EDA Sensing on Key Body Locations. In Proceedings of the 2016 CHI Conference on Human Factors in Computing Systems (CHI '16). ACM, New York, NY, USA, 5665-5674. DOI : http://dx.doi.org/10.1145/2858036.2858536 
[58] Muhammad Umair, Muhammad Hamza Latif, and Corina Sas. 2018. Dynamic Displays at Wrist for Real Time Visualization of Affective Data. In Proceedings of the 2018 ACM Conference Companion Publication on Designing Interactive Systems (DIS '18 Companion). ACM, New York, NY, USA, 201-205. DOI : http://dx.doi.org/10.1145/3197391.3205436

[59] Muhammad Umair, Corina Sas, and Muhammad Hamza Latif. 2019. Towards Affective Chronometry: Exploring Smart Materials and Actuators for Real-time Representations of Changes in Arousal. In Proceedings of the 2019 on Designing Interactive Systems Conference (DIS '19). ACM, New York, NY, USA, 1479-1494. DOI :

http://dx.doi.org/10.1145/3322276. 3322367

[60] Elsa Vaara, Iuliana Silvundefinedundefinedan, Anna Ståhl, and Kristina Höök. 2010. Temporal Relations in Affective Health. In Proceedings of the 6th Nordic Conference on Human-Computer Interaction: Extending Boundaries (NordiCHI'10). ACM, New York, NY, USA, 833-838. DOI : http://dx.doi.org/10.1145/1868914.1869043

[61] Anna Vallgårda and Johan Redström. 2007. Computational Composites. In Proceedings of the SIGCHI Conference on Human Factors in Computing Systems (CHI '07). ACM, New York, NY, USA, 513-522. DOI: http://dx.doi.org/10.1145/1240624.1240706

[62] Mikael Wiberg. 2013. Methodology for materiality: interaction design research through a material lens. Personal and Ubiquitous Computing 18, 3 (May 2013), 625-636. DOI :

http://dx.doi.org/10.1007/s00779-013-0686-7
[63] Danielle Wilde, Anna Vallgårda, and Oscar Tomico. 2017. Embodied Design Ideation Methods: Analysing the Power of Estrangement. In Proceedings of the 2017 CHI Conference on Human Factors in Computing Systems - CHI '17. ACM Press, Denver, Colorado, USA, 5158-5170. DOI :

http://dx.doi.org/10.1145/3025453.3025873

[64] Graham Wilson, Dobromir Dobrev, and Stephen A. Brewster. 2016. Hot Under the Collar: Mapping Thermal Feedback to Dimensional Models of Emotion. In Proceedings of the 2016 CHI Conference on Human Factors in Computing Systems (CHI '16). ACM, New York, NY, USA, 4838-4849. DOI : http://dx.doi.org/10.1145/2858036.2858205

[65] Charles Windlin, Anna Ståhl, Pedro Sanches, Vasiliki Tsaknaki, Pavel Karpashevich, Madeline Balaam, and Kristina Höök. 2019. Soma Bits: Mediating technology to orchestrate bodily experiences. RTD Conference March (2019), 19-22. DOI : http://dx. doi .org/10.6084/m9. figshare.7855799.v2

[66] Ludwig Wittgenstein, G. E. M Anscombe, G. H. von Wright, and Heikki Nyman. 1988. Remarks on the philosophy of psychology. University of Chicago Press. OCLC: 25540686.

[67] Xiao Zhang and Ron Wakkary. 2014. Understanding the Role of Designers' Personal Experiences in Interaction Design Practice. In Proceedings of the 2014 Conference on Designing Interactive Systems (DIS '14). ACM, New York, NY, USA, 895-904. DOI :

http://dx.doi.org/10.1145/2598510.2598556 